\title{
MUCINOUS ADENOCARCINOMA MIMICKING CRYPTOGENIC ORGANIZING PNEUMONIA: A CASE REPORT
}

\author{
Barış Altunel ${ }^{1}$ (D), Müçteba Gökalp Özer ${ }^{1}$ (D), Aykut Alkan² \\ ${ }^{1}$ Trakya University School of Medicine, Edirne, TURKEY \\ ${ }^{2}$ Trakya University School of Medicine, Department of Radiology, Edirne, TURKEY
}

\section{ABSTRACT}

Mucinous adenocarcinoma of the lung is a type of malignancy that originates from glands or gland-like structures in the lung and can mimic pneumonia forms on radiological images. Our case report aims to raise awareness about this radiological pattern. A 72-year-old male patient was applied to an external center with the complaint of cough with sputum and back pain. Computed tomography revealed a kind of consolidation in the lower right lung zone of the patient. The patient, whose consolidation progressed in the lower right zone of the lung, was diagnosed with cryptogenic organizing pneumonia, and then corticosteroids were started. His condition worsened and a biopsy was requested for further investigation. The biopsy revealed mucinous adenocarcinoma of the lung in the patient. Mucinous adenocarcinoma is a malignancy that can be misdiagnosed as organizing pneumonia due to the similarities in patterns between the radiological images. Therefore, differential diagnosis of adenocarcinoma should be considered in cases of organizing pneumonia.

Keywords: Neoplasms, pneumonia, mucinous adenocarcinoma

\section{INTRODUCTION}

Lung cancer is one of the world's leading causes of death (1). Lung cancers are mostly divided into two main groups: small cell lung cancers in about $15 \%$ of all lung neoplasms and nonsmall cell lung cancers (NSCLCs) in about $85 \%$ of all lung neoplasms (2). Lung adenocarcinoma is the most common form among non-small cell cancers at about $50 \%$ of all NSCLCs (3). Adenocarcinoma is a malignancy that originates from glands or gland-like structures and can occur in many organs. Pneumonia is the inflammation of the distal lung structures. If the pneumonia is not resolved, it can lead to the organization of inflammatory exudate, which leads to fibrosis in the lung tissue, called organizing pneumonia. Organizing pneumonia is a prominent inflammatory lung disease due to easily reversible intra-alveolar fibrosis $(4,5)$.

Exclusion of any other etiology and a histopathologic examination of the lung tissue leads to the diagnosis of "cryptogenic" organizing pneumonia (COP) while organizing pneumonia secondary to other conditions such as infectious agents, drugs (5-aminosalicylic acid, acebutolol, amiodarone, amphotericin B, bleomycin, busulfan, carbamazepine, cephalosporin, fluvastatin, gold salts, methotrexate, etc.), radiation, or malignancy termed as "secondary" organizing pneumonia (5). Lung adenocarcinoma is known to mimic different types of pneumonia, including organizing pneumonia, and can be confused on computed tomography (CT) imaging (6).

In this study, we aimed to present a patient whose pneumonia mimicked COP, who was later diagnosed with mucinous adenocarcinoma after his condition worsened. As it can be difficult to distinguish between these two pathologies, we believe that this study will raise awareness about the differential diagnosis of these conditions.

Address for Correspondence: Barış Altunel, Trakya University School of Medicine, Edirne, TURKEY

e-mail: baris_altunel@hotmail.com

ORCID iDs of the authors: BA: 0000-0002-4962-8865; MGÖ: 0000-0002-4592-5491; AA: 0000-0001-6903-5314

Received: 04.08.2021 Accepted: 07.09.2021

Cite this article as: Altunel B, Özer MG, Alkan A. Mucinous adenocarcinoma mimicking cryptogenic organizing pneumonia:

A case report. Turk Med Stud J 2022;9(1):25-8.

Copyright@Author(s) - Available online at https://www.turkmedstudj.com/ 


\section{CASE REPORT}

A 72-year-old male patient applied to an external center with complaints of cough with sputum and back pain. The patient was a retired metal worker with a 40-year smoking history and had quit smoking for 12 years. The patient had no chronic disease other than hypothyroidism.

A CT scan was performed on the patient (Figure 1), and the patient who was diagnosed with community-acquired pneumonia was referred to Trakya University School of Medicine Hospital, Department of Chest Diseases for further examination. The chest CT performed on the patient showed pneumonic infiltrates that progressed in the right lower lobe (Figure 2). Flexible bronchoscopy was requested to find the cause of pneumonia. According to the results of bronchoscopy, no signs of infection or endobronchial lesion were found. The basal segment openings of the lung were edematous and bronchoalveolar lavage from the right lower

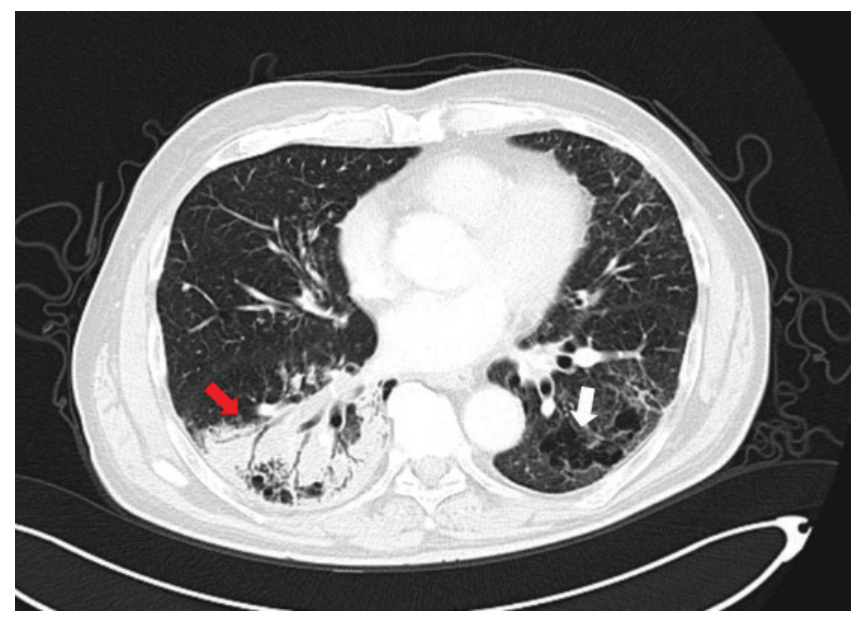

Figure 1: A homogeneous consolidation area with air bronchogram is observed in the posterobasal segment of the lower lobe of the right lung (red arrow). In addition, cystic emphysematous changes are observed in the lower lobe of the left lung (white arrow).

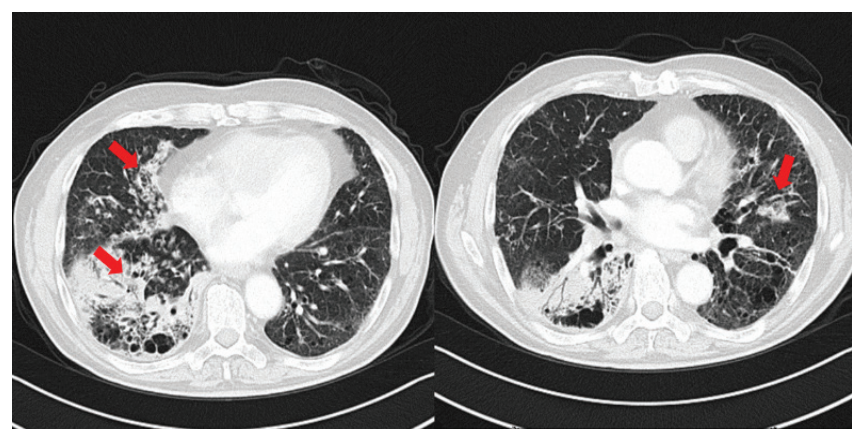

Figure 2: Axial plane contrast computed tomography scan 6 weeks after the first admission. In addition to previous consolidations in the lower right basal segment, newly developed air bronchograms and consolidation areas are observed in both lungs (red arrows). lobe revealed inflammation, but no malignancy was found. In addition, a sample was taken for bronchoalveolar lavage culture.

The patient was then recalled with the results of the lavage culture. The patient did not have chest pain or hemoptysis, but sputum and dyspnea continued. Inspiratory sounds were coarse, and crackles were heard in the lower zone of the right lung. Saturation of peripheral oxygen $\left(\mathrm{SpO}_{2}\right)$ levels were normal. No bacterial growth was detected in the bronchoalveolar lavage culture, and a contrast-enhanced CT scan was requested.

Computed tomography findings (Figure 3) were evaluated as COP due to the absence of other etiological factors. The patient was started on methylprednisolone (40 mg/day) and levofloxacin (750 mg/day) for two weeks. No disease progression or improvement in his condition was observed in this period, and the patient started to suffer from severe dysnepea. The patient was hypoxic with $\mathrm{SpO}_{2}$ levels between $65-70 \%$. The patient was given oxygen supplementation and was started on piperacillin-tazobactam for two weeks. Chest CT was also requested. Both lungs had peripheral consolidations compared to older CT scans (Figure 4). The patient also had a negative coronavirus disease-2019 polymerase chain reaction test in this period.

The patient was then referred to the department of interventional radiology for percutaneous transthoracic lung

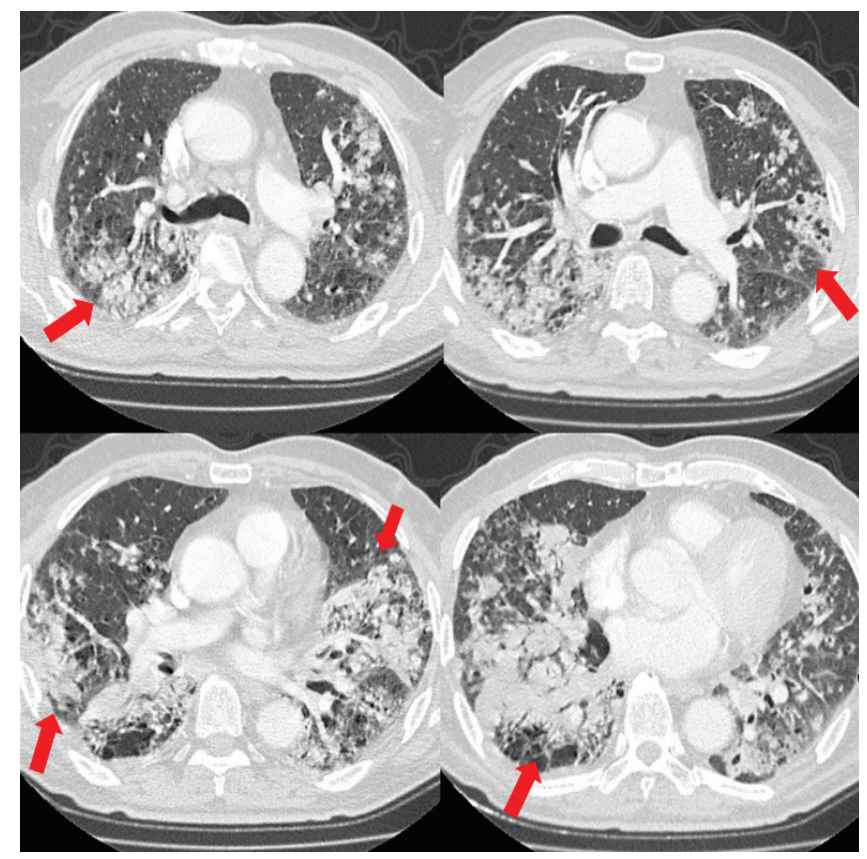

Figure 3: Axial plane contrast computed tomography scan, 5 months after the first admission. The increasing number of infiltration areas are observed in both lungs (red arrows). Infiltration areas consist of nodular consolidation that tends to coalesce. 


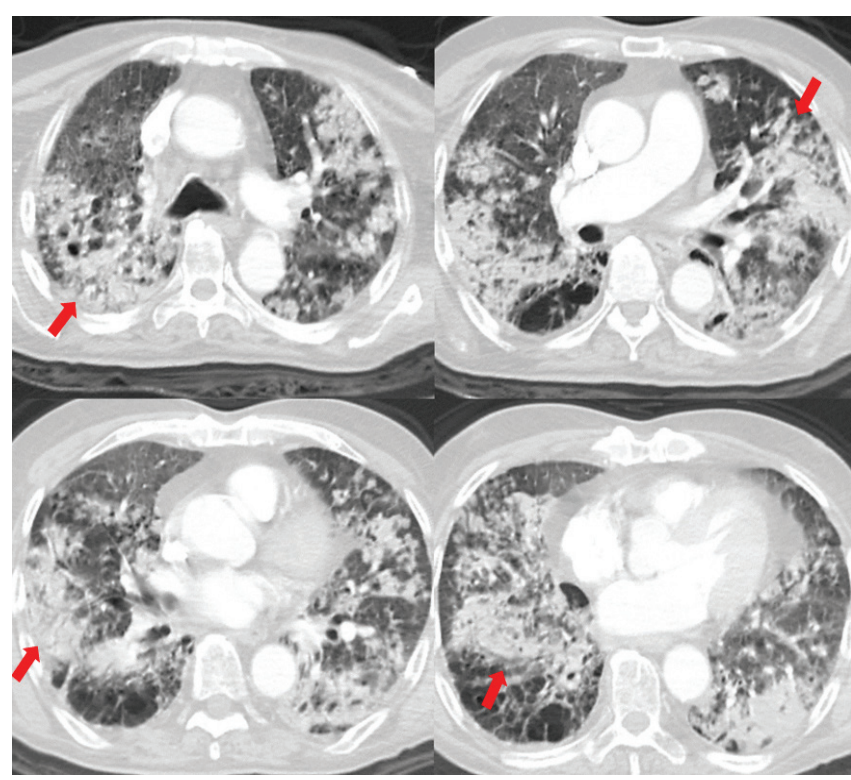

Figure 4: Axial plane contrast computed tomography scan, 7 months after the first admission. Increasing infiltration areas are observed in both lungs. Infiltration areas consist of common consolidation areas (red arrows).

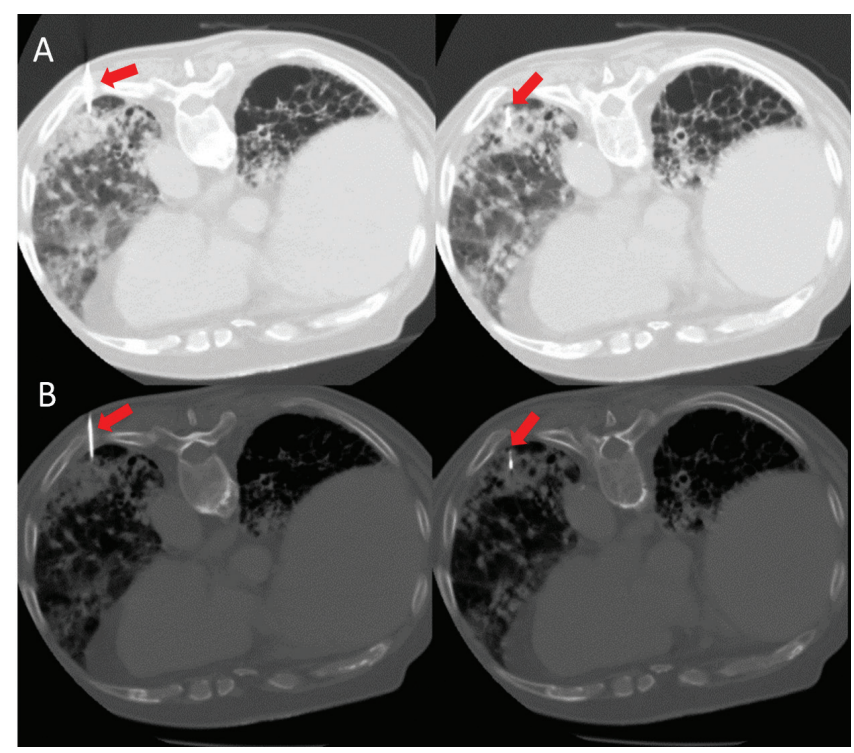

Figure 5: Core needle biopsy was performed by entering percutaneous from the consolidation area observed in the lower lobe of the left lung (red arrows). A: Lung parenchyma window. B: Bone window.

biopsy (Figure 5). Biopsy material was positive for thyroid transcription factor- 1 and negative for P40 stains, confirming the diagnosis of lung adenocarcinoma (Figure 6, 7).

Adenocarcinoma was determined as mucinous type with lepidic pattern [World Health Organization (WHO) classification of bronchoalveolar carcinoma pre-2011]. Although a definite classification of invasiveness could not be made on the small biopsy material after diagnosis, the

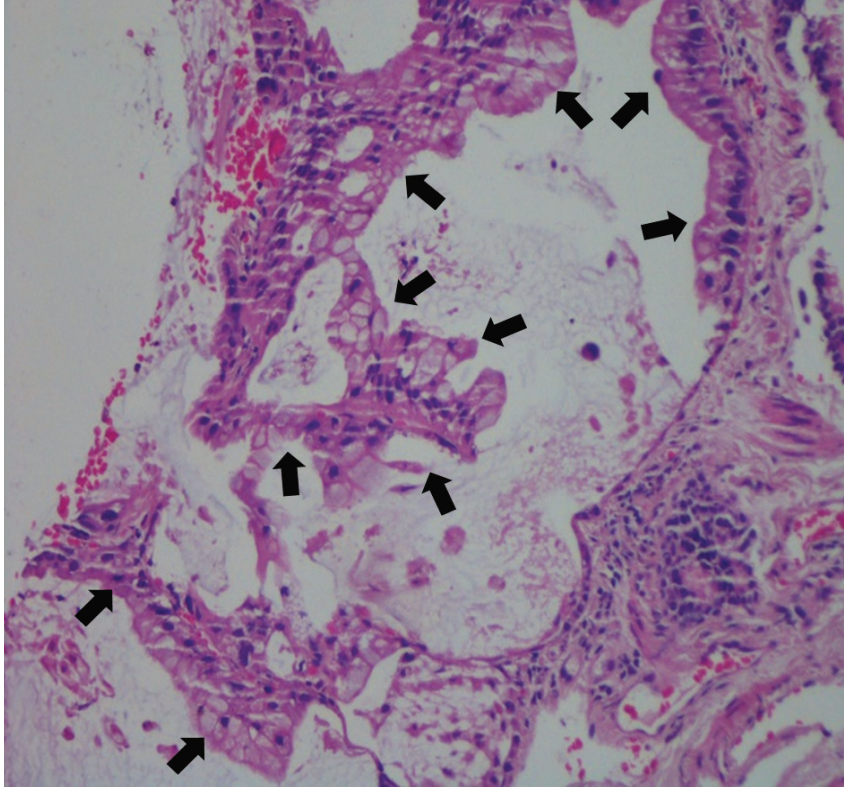

Figure 6: Cubic and columnar tumor cells with a basally located nucleus and abundant cytoplasmic mucin lining the alveolar wall (arrows) (hematoxylin \& eosin stain, x100 magnification).

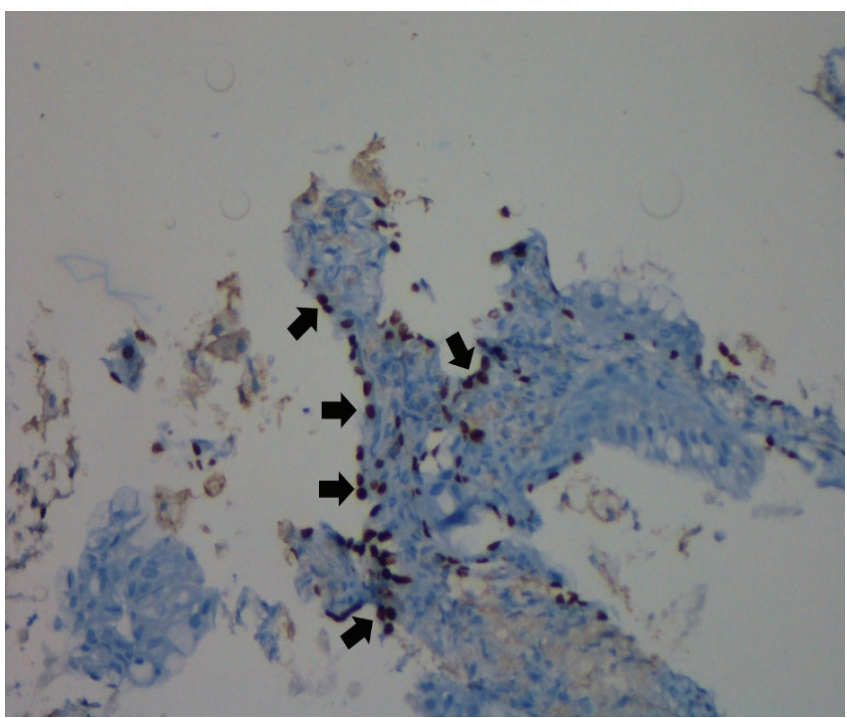

Figure 7: Positive stain for thyroid transcription factor-1 antibody in pneumocyte nuclei (arrows) (x100 magnification).

patient was referred to the department of medical oncology for chemotherapy.

\section{DISCUSSION}

Cancer is among the deadliest diseases with approximately 10 million deaths worldwide (7). According to studies conducted by $\mathrm{WHO}$, lung cancer ranks first in the list with 1.8 million deaths in 2020 (7). 
Adenocarcinoma is the most common histological subtype of lung cancer, and many different subtypes are depending on the mucinous differentiation and invasion stage (3). In our case, the patient had mucinous adenocarcinoma with a possible lepidic predominant pattern.

World Health Organization 2011 classification removed the term of bronchoalveolar carcinoma and added subtypes of adenocarcinoma in situ, minimally invasive adenocarcinoma, invasive mucinous adenocarcinoma, and lepidicpredominant invasive non-mucinous adenocarcinoma (6). Organizing pneumonia CT patterns include peripheric patchy consolidations, ground-glass opacities, perilobular opacities, reversed halo sign, nodules, and masses (8). Consolidations and ground-glass opacities were found to be characteristics of both general lung adenocarcinoma (previously bronchoalveolar carcinoma) and organizing/ infectious pneumonia, though the peripheral distribution of consolidation and nodular pattern were found to be significantly more common in patients with adenocarcinoma $(8,9)$. Findings of cysts, cavities, CT angiogram sign, and leafless tree bronchogram sign were not significantly different among pneumonia and adenocarcinoma (9).

Detterbeck et al. (10) described pneumonic type adenocarcinoma radiologically as regional rather than the nodular appearance of varying areas of ground glass and consolidation. Microscopically they are typically found to be mucinous adenocarcinomas that are invasive with a predominance of lepidic growth. The increasing prevalence of CT imaging made cancers with ground glass and/or lepidic nodules seen more commonly. Moderate levels of correlation have been reported between imaging and histological subtypes of these pneumonic-type adenocarcinomas. The consolidative pattern was around $33-75 \%$ of the cases. Areas of ground glass are observed in about $75 \%$ of the cases (10). The five-year disease-free survival rate for lepidic predominant adenocarcinoma was found to be around $72-90 \%$ (11).

In our case, we observed a similar progressive consolidation pattern on CT scans of the patient, and the peripheral consolidations suggested the diagnosis of lung adenocarcinoma. However, the absence of nodular pattern was more in favor of pneumonia. It can be confused with mucinous adenocarcinoma of the lung and COP due to similar radiological findings. Other diagnoses must be ruled out before a diagnosis of COP can be made.

Acknowledgments: We would like to thank Osman Kula and Fethi Emre Ustabaşıoğlu from Department of Radiology, Bilkay Serez from Department of Chest Diseases, Trakya University School of Medicine for their guidance and support in preparing this manuscript. We also would like to thank
Merve Çimen from the Department of Pathology, Trakya University School of Medicine, for providing pathology images and their interpretation.

\section{Ethics Committee Approval: N/A}

Informed Consent: Informed verbal consent was obtained from the patient.

Conflict of Interest: The authors declared no conflict of interest.

Author Contributions: Concept: B.A., M.G.Ö., A.A., Design: B.A., M.G.Ö., A.A., Supervision: B.A., M.G.Ö., A.A., Resources: A.A., Materials: A.A., Data Collection and/or Processing: B.A., M.G.Ö., A.A., Analysis and/or Interpretation: B.A., M.G.Ö., A.A., Literature Search: B.A., M.G.Ö., A.A., Writing Manuscript: B.A., M.G.Ö., A.A., Critical Review: B.A., M.G.Ö., A.A.

Financial Disclosure: The authors declared that this study received no financial support.

\section{REFERENCES}

1. Global Burden of Disease Cancer Collaboration, Fitzmaurice C, Dicker D, Pain A et al. The Global Burden of Cancer 2013. JAMA Oncol 2015;1:50527. [Crossref]

2. Herbst RS, Heymach JV, Lippman SM. Lung cancer. N Engl J Med 2008;359:1367-80. [Crossref]

3. Travis WD, Brambilla $E$, Noguchi $M$ et al. International association for the study of lung cancer/american thoracic society/european respiratory society international multidisciplinary classification of lung adenocarcinoma. J Thorac Oncol 2011;6:244-85. [Crossref]

4. Sulavik SB. The concept of "organizing pneumonia". Chest 1989;96:9679. [Crossref]

5. Cordier JF. Cryptogenic organising pneumonia. Eur Respir J 2006;28:42246. [Crossref]

6. Pascoe HM, Knipe HC, Pascoe D, et al. The many faces of lung adenocarcinoma: A pictorial essay. J Med Imaging Radiat Oncol 2018;62:654-61. [Crossref]

7. World Health Organization. Cancer (serial online) (cited 2021 July 7). Available from: URL: http://www.who.int/news-room/fact-sheets/ detail/cancer. [Crossref]

8. Zare Mehrjardi M, Kahkouee S, Pourabdollah M. Radio-pathological correlation of organizing pneumonia (OP): a pictorial review. Br J Radiol 2017;90:20160723. 2017 Feb 17. doi: 10.1259/bjr.20160723. [Epub ahead of print] [Crossref]

9. Aquino SL, Chiles C, Halford P. Distinction of consolidative bronchioloalveolar carcinoma from pneumonia: do CT criteria work? AJR Am J Roentgenol 1998;171:359-63. [Crossref]

10. Detterbeck FC, Marom EM, Arenberg DA et al. The IASLC Lung Cancer Staging Project: Background Data and Proposals for the Application of TNM Staging Rules to Lung Cancer Presenting as Multiple Nodules with Ground Glass or Lepidic Features or a Pneumonic Type of Involvement in the Forthcoming Eighth Edition of the TNM Classification. J Thorac Oncol 2016;11:666-80. [Crossref]

11. Suh JH. Current readings: pathology, prognosis, and lung cancer. Semin Thorac Cardiovasc Surg 2013;25:14-21. [Crossref] 